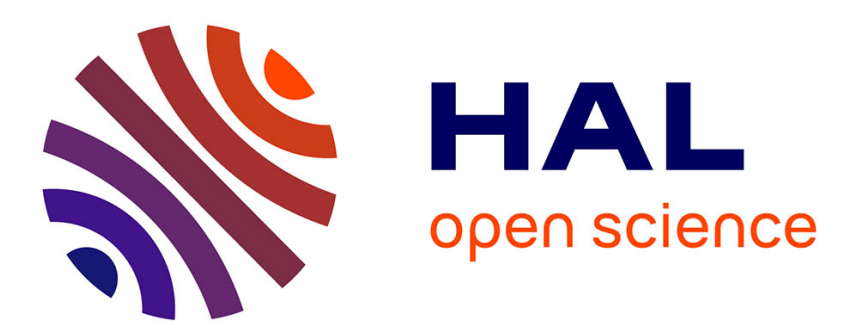

\title{
Simulation numérique et observation de l'écoulement d'une suspension de fibres dans une filière à géométrie complexe
}

\author{
Kunji Chiba, Francisco Chinesta
}

\section{- To cite this version:}

Kunji Chiba, Francisco Chinesta. Simulation numérique et observation de l'écoulement d'une suspension de fibres dans une filière à géométrie complexe. Revue des composites et des matériaux avancés $=$ Journal of Composite and Advanced Materials, 2002, 12 (3), pp.499-513. 10.3166/rcma.12.499-513 . hal-00020736

\section{HAL Id: hal-00020736 https://hal.science/hal-00020736}

Submitted on 17 Feb 2018

HAL is a multi-disciplinary open access archive for the deposit and dissemination of scientific research documents, whether they are published or not. The documents may come from teaching and research institutions in France or abroad, or from public or private research centers.
L'archive ouverte pluridisciplinaire HAL, est destinée au dépôt et à la diffusion de documents scientifiques de niveau recherche, publiés ou non, émanant des établissements d'enseignement et de recherche français ou étrangers, des laboratoires publics ou privés. 


\title{
Simulation Numérique et Observation de l'Ecoulement d'une Suspension de Fibres dans une Filière à Géométrie Complexe
}

\section{${\text { Kunji } \text { Chiba }^{1} \text { et Francisco Chinesta }}^{2}$}

\author{
${ }^{1}$ Faculty of Education, Shiga University \\ 2-5-1 Hiratsu, Otsu, Shiga 520-0862, Japan \\ kchiba@sue.shiga-u.ac.jp \\ ${ }^{2}$ LMSP: UMR CNRS - ENSAM - ESEM \\ 151 Boulevard de l’Hôpital, F-75013 Paris, France. \\ francisco.chinesta@paris.ensam.fr
}

\begin{abstract}
RESUME. Ce travail porte sur la simulation numérique et l'observation de l'orientation des fibres d'une suspension dans une géométrie complexe. Les fibres sont supposées rigides et le fluide est supposé Newtonien. Les problèmes traités dans cet article seront: (i) écoulement d'une suspension diluée dans une contraction axisymétrique 4:1 où l'hypothèse d'alignement local des fibres avec l'écoulement sera introduite dans le calcul, dont les résultats seront confrontés à des observations expérimentales; (ii) résolution numérique de l'écoulement dans une filière plate, où l'on considère couplées la cinématique de l'écoulement et l'orientation $3 D$ des fibres avec différents facteurs de forme. Le calcul sera effectué à l'aide d'une méthode statistique, qui s'avère adaptée à ce type de calcul, par rapport aux autres approches basées sur le calcul de la fonction de distribution des orientations ou sur la résolution des équations gouvernant l'évolution des différents tenseurs d'orientation, et qui impliquent l'utilisation d'une relation de fermeture, dont la validité est remise en question dans certains cas.
\end{abstract}

MOTS CLES: suspensions fibres courtes, méthode statistique, écoulement en contraction, orientation des fibres 


\section{Introduction}

Les suspensions de fibres présentent souvent une anisotropie induite par l'écoulement. L'addition de fibres dans un fluide Newtonien, même en faible quantité, peut changer radicalement son comportement en écoulement. Ces dernières années, l'importance du couplage entre la cinématique de l'écoulement et l'orientation des fibres a été reconnue (Rosenberg et al., 1990; Phan-Thien et Graham 1991; Ranganathan et Advani, 1993; Altan et Rao, 1995; Ahmed et Alexandrou, 1994; Chung et Kwon, 1995; Lipscomb et al., 1988; Chiba et al. 1990; Baloch et Webster, 1995; Ausias et al., 1992; Ghosh et al., 1995). De plus, l'orientation des fibres détermine les propriétés mécaniques des composites à fibres courtes et sa détermination devient aujourd'hui un point clé dans la simulation des procédés de fabrication.

Chiba et al., (2001) ont étudié l'écoulement 2D (dans une filière plate) d'une suspension de fibres courtes dans un fluide Newtonien en couplant la cinématique de l'écoulement avec une distribution de l'orientation des fibres considérée plane. L'orientation d'une grande quantité de fibres a été calculée à partir de l'intégration de l'équation de Jeffery sur différentes lignes de courant. Cette méthode statistique s'est avéré être une alternative au calcul de la distribution de l'orientation des fibres en résolvant l'équation de Fokker-Planck, ou à l'intégration des équations d'évolution des tenseurs d'orientation qui nécessitent l'introduction d'une relation de fermeture appropriée.

Ce travail porte sur les suspensions de fibres dans des géométries complexes, tant du point de vue de la simulation numérique que de l'observation expérimentale. Les fibres seront supposées rigides en suspension dans un fluide Newtonien. Cet article est structuré en deux parties :

1. Ecoulement dans une contraction circulaire 4:1.

La prédiction du régime d'écoulement utilisant l'hypothèse d'alignement local des fibres avec l'écoulement sera comparée avec les résultats de l'observation expérimentale. Il n'y a pas beaucoup de travaux portant sur les écoulements de suspensions présentant des zones de recirculation. Lipscomb et al. (1988) ont étudié les effets d'entrée d'une suspension de fibres dans un tube. Le model TIF (Transversely Isotropic Fluid), proposé par Ericksen (1960), a été appliqué au cas des suspensions de fibres à rapport de forme élevé. Les résultats obtenus ont été comparés aux observations expérimentales en régime semi-dilué, concernant principalement la croissance de la recirculation, et ils sont très similaires. Dans la simulation, l'hypothèse d'alignement local des fibres avec l'écoulement a été introduite. Baloch et Webster (1995) ont simulé différents écoulements en contraction en utilisant l'équation constitutive de Lipscomb et al. Ils ont comparé l'hypothèse d'alignement local des fibres dans l'écoulement et dans la direction perpendiculaire à l'écoulement, avec les observations expérimentales de Abdul-Karem et al. (1993).

2. Simulation numérique de l'écoulement d'une suspension dans une filière plate, pour des fibres avec différents rapports de forme, où cette orientation est supposée 3D. Dans le cas des fibres à rapport de forme faible, l'effet de la rotation des fibres tout au long de leurs trajectoires sera mis en évidence.

En ce qui concerne le développement du régime d'écoulement d'une suspension dans une filière plate, nous pouvons citer les travaux d'Altan et al. (1992) qui ont supposé l'orientation plane et utilisé le modèle de Dinh-Armstrong (1984). Ils ont résolu l'équation d'évolution du tenseur d'orientation de quatrième ordre avec une relation de fermeture quadratique du tenseur du sixième ordre. Chono et Makino (1995) ont utilisé également le model de Dinh- 
Armstrong et une équation d'évolution du tenseur de déformation équivalente proposée par Lipscomb (1986) dans le cas d'une orientation plane. Ils ont comparé les solutions exactes avec leurs prédictions numériques. Ausias et al. (1994) ont considéré le cas axisymétrique en couplant la cinématique de l'écoulement et l'orientation 3D des fibres dans l'équation constitutive proposée par Lipscomb et al. (1988).

En pratique, des états d'orientation plane sont rarement observés, et même pour un écoulement plan, on peut retrouver des états d'orientation 3D. Dans ce travail nous avons préféré coupler une cinématique plane avec une orientation $3 \mathrm{D}$ pour des fibres à différent rapport de forme. De la même façon, ces simulations nous serviront d'une part à évaluer l'incidence de la rotation continue des fibres à faible rapport de forme sur la cinématique et sur les contraintes, et d'autre part à répondre à la question concernant l'existence d'un régime établi.

\section{Ecoulement d'une suspension diluée dans une contraction circulaire $4: 1$}

\subsection{Modèle mécanique}

Les équations de conservation de la masse et de la quantité de mouvement pour un matériau incompressible s'écrivent :

$$
\begin{aligned}
& \operatorname{Div} \underline{v}=0 \\
& \rho d \underline{v} / d t=-G r a d p+\operatorname{Div} \underline{=}
\end{aligned}
$$

où $\underline{v}$ est le vecteur vitesse, $\rho$ la densité, $p$ la pression, $\underline{\underline{\tau}}$ le tenseur des extra-contraintes et $d / d t$ la dérivée matérielle. La théorie des milieux continus développée par Lipscomb et al. (1988) dans le cadre des suspensions diluées de particules rigides avec un rapport de forme élevé est utilisée pour prendre en compte l'existence des fibres en suspension

$$
\stackrel{\tau}{=}=2 \eta \underline{D}+\phi \mu \underline{D} \underline{\underline{D}} \underset{=}{\stackrel{a}{=}}
$$

où $\eta$ est la viscosité du fluide matrice, supposé Newtonien, $\underline{\underline{D}}$ est le tenseur des taux de déformation, $\phi$ est la fraction (en volume) de fibres, $\mu$ est un paramètre du matériau et $\stackrel{a}{=}$ est le tenseur d'orientation d'ordre 4

$$
\underline{\underline{\underline{a}}}=\oint \underline{p} \otimes \underline{p} \otimes \underline{p} \otimes \underline{p} \psi(\underline{p}) d \underline{p}
$$

où $\underline{p}$ est le vecteur unitaire qui défini l'orientation d'une fibre et $\psi(p)$ est la fonction de distribution de l'orientation des fibres: l'orientation des fibres dans un volume de fluide peut être distribuée. Pour des fibres à rapport de forme élevé (rapport entre la longueur et le diamètre) $\lambda \geq 10, \mu$ vient donnée par (Lipscomb et al., 1988) 
$\mu=\frac{\eta \lambda^{2}}{\ln (\lambda)}$

Les équations précédentes appliquées à l'écoulement dans une contraction de rapport 4:1 peuvent être résolues dans le cas d'un alignement des fibres avec l'écoulement, $\underline{p}=\underline{v} /\|\underline{v}\|$, sans avoir à considérer la distribution des orientations.

Nous étudions la validité de l'hypothèse d'alignement. La figure 1 montre les résultats observés expérimentalement dans une contraction plane à rapport $3: 1$. Les fibres sont bien alignées partout dans le domaine dans le cas où l'on considère un fluide à forte élasticité (polyacrylamide -PAA- en solution aqueuse), ce qui est loin d'être le cas pour un solvant Newtonien (figure 1 (a)). Cependant, dans la partie convergente de l'écoulement, la validité de l'hypothèse semble être confirmée dans tous les cas.

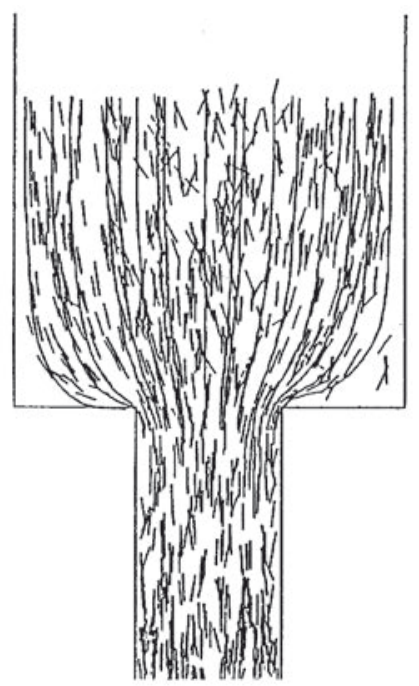

(a)

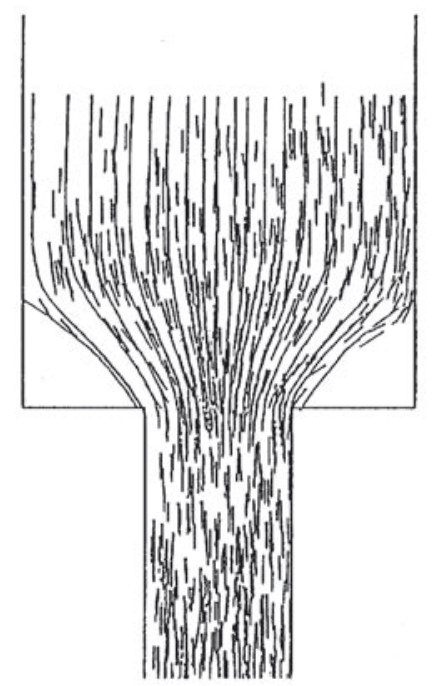

(b)

Figure 1. Orientation observée des fibres dans une contraction plane $3: 1(40 \mathrm{~mm} \times 300 \mathrm{~mm}$ en entrée); (a) eau, débit $\mathrm{Q}=206.7 \mathrm{~cm}^{3} / \mathrm{s}$; (b) solution aqueuse de PAA, $0.2 \%$ en mass, $\mathrm{Q}=207.6$ $\mathrm{cm}^{3} / \mathrm{s}$.

Les équations de mouvement ont été exprimées avec les variables fonction de courant et vorticité, et résolues à l'aide de la méthode des différences finies. La taille du maillage est de 0.25 dans les deux directions (axiale et radiale) normalisées avec le diamètre du tube d'entrée. Le paramètre $\phi \mu / \eta=\phi \lambda^{2} / \ln (\lambda)$ a été variable afin d'étudier son incidence sur la cinématique de l'écoulement.

\subsection{Changement de régime de l'écoulement}

Le tube circulaire utilisé pour la visualisation expérimental mesure $32 \mathrm{~mm}$ de diamètre et $500 \mathrm{~mm}$ de long, contenant une contraction à rapport 4:1. Le fluide a été chargé avec des particules de polystyrène d'un diamètre inférieur à $0.1 \mathrm{~mm}$ et un faisceau plan de lumière a été dirigé sur le plan central de l'écoulement. La suspension est composée de fibres vinyliques de diamètre $d=13 \mu \mathrm{m}$ et de longueur $l=3 \mathrm{~mm}(\lambda=231)$, et d'un mélange de sirop 
de maïs $\left(65 \%\right.$ en masse) et d'eau, avec une viscosité $\eta=0.08 \mathrm{~Pa} \cdot \mathrm{s}$ à $16.5^{\circ} \mathrm{C}$. Les lignes de courant calculées numériquement à très faibles nombres de Reynolds sont illustrées dans la figure 2 dans le cas d'un fluide Newtonien et dans le cas d'une suspension avec $\phi \mu / \eta=8$. Nous pouvons constater que la taille de la recirculation augmente considérablement dans le cas de la suspension, même si une valeur $\phi \mu / \eta=8$ correspond à une très faible concentration en volume de fibres $(0.082 \%)$.

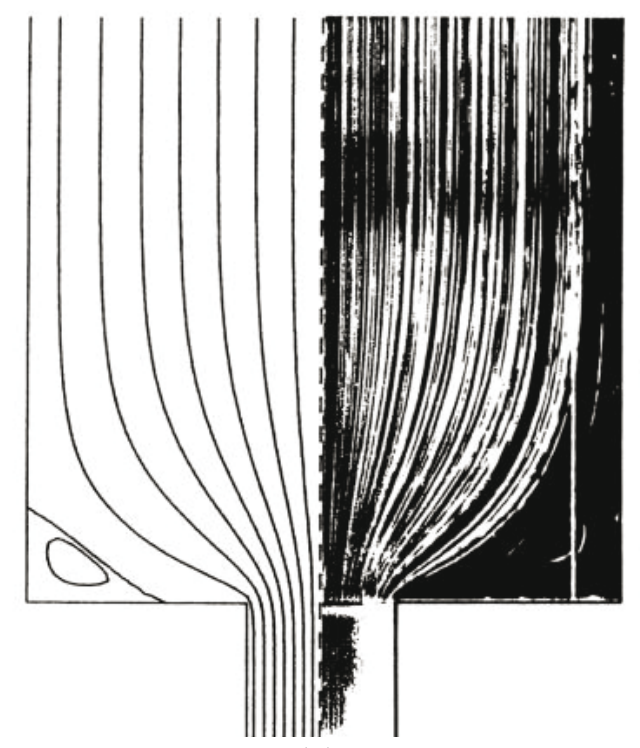

(a)

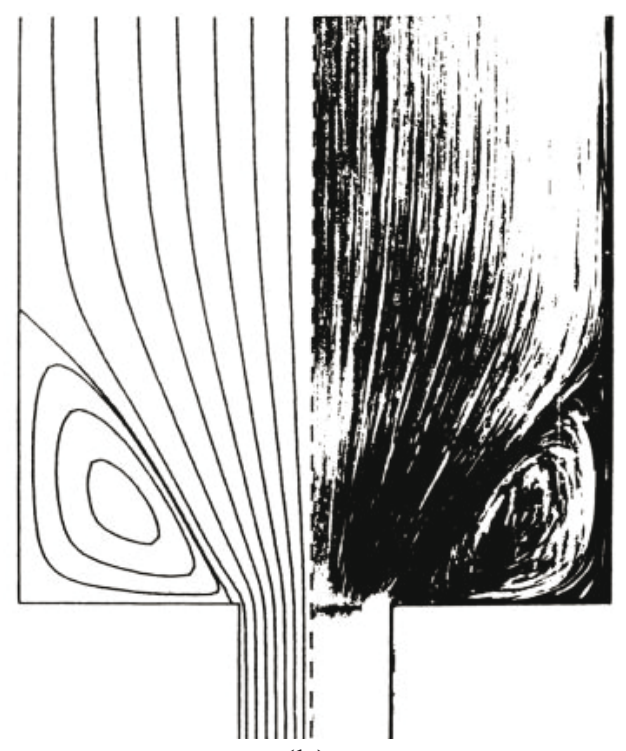

(b)

Figure 2. Lignes de courant calculées et observées dans une contraction circulaire à rapport $4: 1$ à très faibles nombres de Reynolds; (a) fluide Newtonien avec $\operatorname{Re}=0.01$ dans le calcul et $\operatorname{Re}=0.29$ dans l'observation; (b) suspension de fibres avec $\phi \mu / \eta=8$.

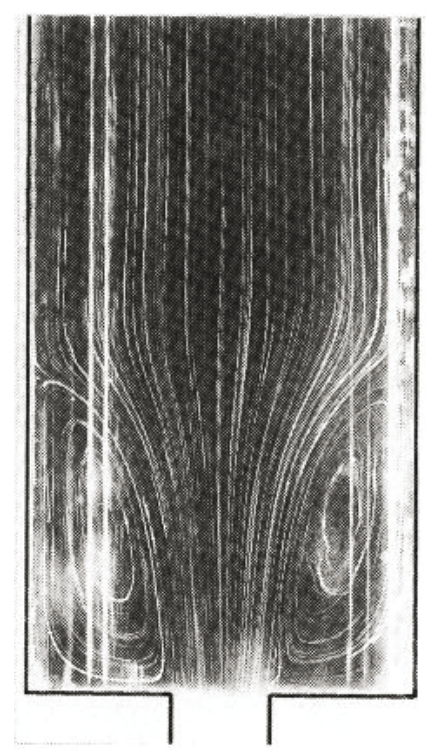

Figure 3. Lignes de courant observées expérimentalement pour une solution aqueuse de PAA $(0.1 \%$ en masse $)$ dans une contraction circulaire à rapport $4: 1(\mathrm{Re}=0.37)$. 
L'écoulement observé présente de notables différences par rapport à la recirculation convexe caractéristique des systèmes comportant des molécules flexibles, comme on peut l'apprécier dans la figure 3 .

Le calcul, tant comme l'observation, ont prouvé que la taille de la recirculation augmente avec la concentration volumique de fibres et/ou avec le rapport de forme des fibres. L'incidence du nombre de Reynolds peut alors être mise en évidence dans la figure 4 : l'écoulement principal pousse la zone de recirculation vers le coin de la contraction, et en même temps, les lignes de courant de l'écoulement principal deviennent concaves.

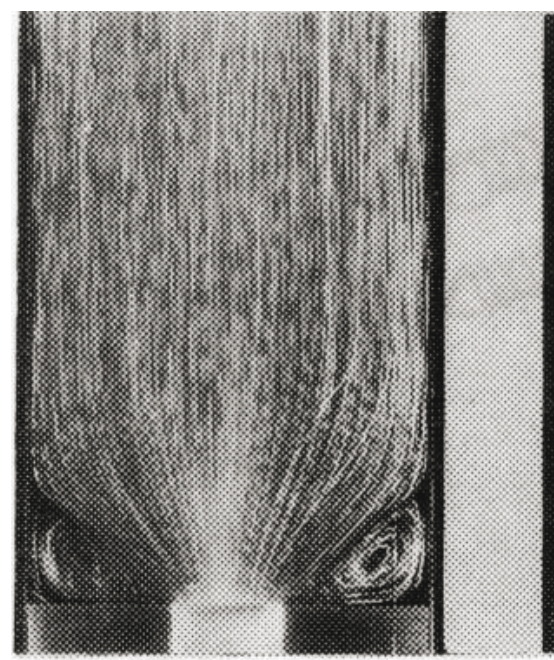

(a)

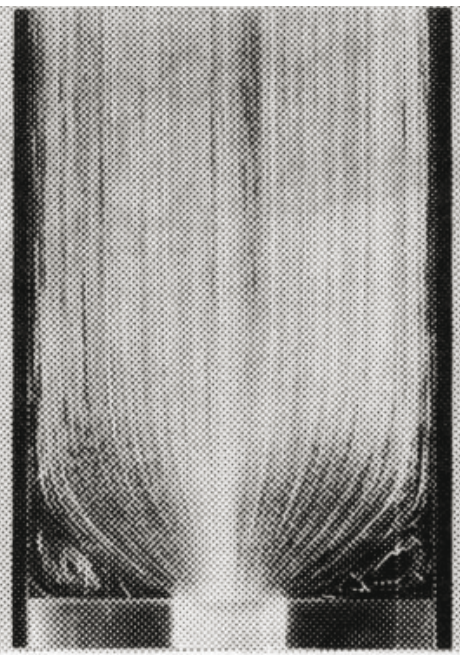

(b)

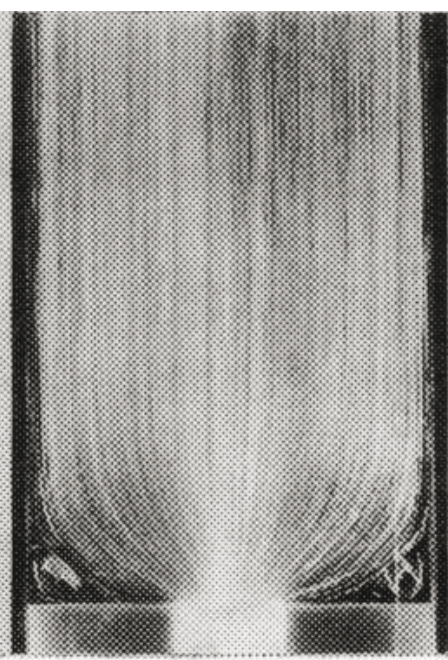

(c)

Figure 4. Visualisation de l'écoulement pour une suspension caractérisée par $\phi \mu / \eta=4$ dans une contraction circulaire 4:1; (a) $\operatorname{Re}=0.28$; (b) $\operatorname{Re}=1.14$; (c) $\operatorname{Re}=2.26$.

\subsection{Champ de contraintes dans l'écoulement d'un fluide Newtonien et d'une suspension de fibres courtes}

La figure 5 représente la distribution de la différence de contraintes normales $\tau_{z z}^{*}-\tau_{r r}^{*}$ pour $\operatorname{Re}=0.01$ : (a) est la solution dans l'écoulement Newtonien et (b) est la solution pour la suspension caractérisée par $\phi \mu / \eta=8$. Les axes z et $r$ représentent l'axe de l'écoulement et la direction radiale respectivement; les composantes de la contrainte ont été normalisées selon $\tau_{i j}^{*}=\tau_{i j} /(2 \eta U / D)$. Dans l'entrée de la contraction, le fluide est fortement étiré tout au long de l'axe de l'écoulement, et la différence de contraintes $\tau_{z z}^{*}-\tau_{r r}^{*}$ devient maximale à proximité de la contraction. La valeur maximale de $\tau_{z z}^{*}-\tau_{r r}^{*}$ est d'environ 200 dans le cas Newtonien, et elle devient d'environ 700 pour la suspension. Ainsi, l'addition de fibres courtes, même à très faible concentration, dans un fluide Newtonien change radicalement la différence de contraintes normales. De l'autre coté, la contrainte de cisaillement reste similaire dans les deux cas. 


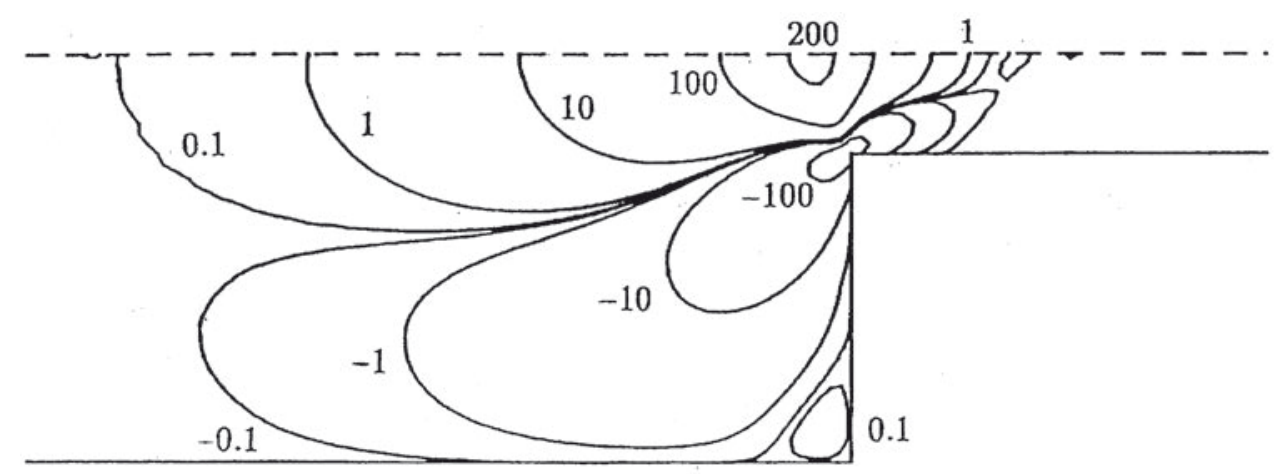

(a)

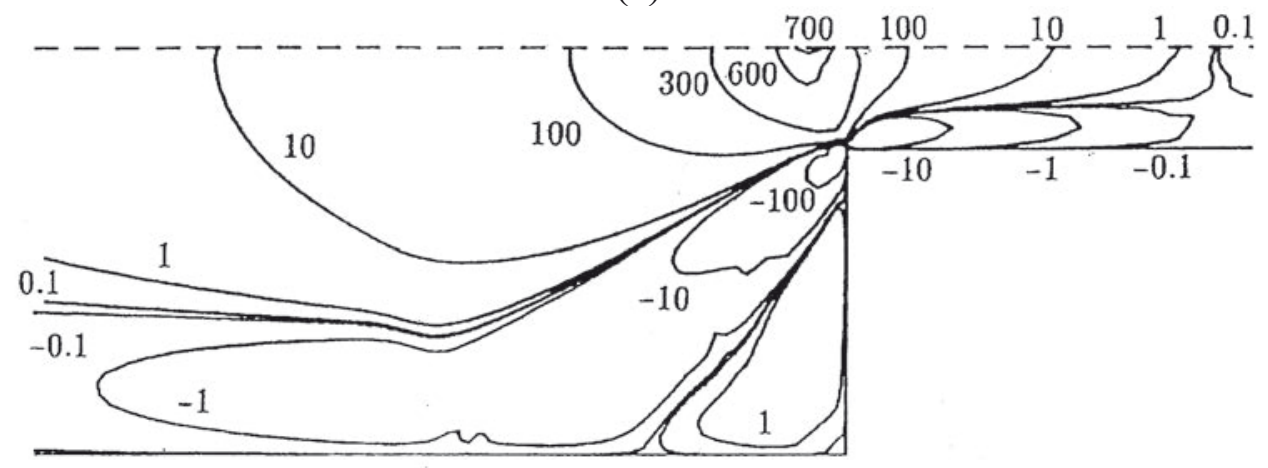

(b)

Figure 5. Différence de contraintes normales $\tau_{z z}^{*}-\tau_{r r}^{*}$ dans une contraction circulaire 4:1 ( $\operatorname{Re}=0.01)$ : (a) fluide Newtonien; (b) suspension de fibres avec $\phi \mu / \eta=8$.

\subsection{Conclusions}

Nous venons de décrire les résultats numériques et les observations expérimentales concernant l'écoulement en contraction des suspensions diluées de fibres courtes rigides à fort rapport de forme. Le principal résultat concerne l'augmentation de la taille de la zone recirculante, ainsi que la très forte augmentation de la différence des contraintes normales. L'écoulement principal devient conique dans le cas des suspensions, ce qui n'est pas le cas des systèmes composés de molécules flexibles, tels que les solutions de PAA.

\section{Solution numérique de l'écoulement d'une suspension de fibres dans une filière plate}

Le modèle couplé d'écoulement (cinématique - orientation des fibres 3D) sera résolu dans le domaine associé à une filière plate de section rectangulaire. Même pour cette cinématique plane (définie dans le plan x-y), nous verrons que l'orientation est, en effet, 3D. Différents champs (vitesse, orientation, contraintes, ...) seront obtenus pour des fibres à rapport de forme infini et fini. 


\subsection{Schéma statistique}

L'évolution du vecteur unitaire qui définit l'orientation des fibres $p$ en suspension dans un fluide Newtonien est décrite par l'équation de Jeffery :

$\frac{d \underline{p}}{d t}=\underline{\underline{\Omega}} \underline{p}+k\left(\underline{\underline{D}}-\underline{p}^{T} \underline{\underline{D}} \underline{p}\right) \underline{p}$

où le paramètre $k$ dépend du rapport de forme des fibres

$k=\left(\lambda^{2}-1\right) /\left(\lambda^{2}+1\right)$

Le tenseur d'orientation de second ordre $\underset{=}{a}$, défini par l'équation [8], est introduit pour décrire de forme concise la distribution de l'orientation des fibres. Ainsi, l'état d'orientation peut être illustré par une ellipsoïde dont la longueur et l'orientation des axes sont donnés par les valeurs propres et les directions principales du tenseur $\underset{=}{a}$. Nous pouvons donc affirmer que la longueur de ces axes indique le degré d'orientation dans la direction correspondante.

$\underline{\underline{a}}=\oint \underline{p} \otimes \underline{p} \psi(\underline{p}) d \underline{p}$

Nous utilisons un schéma statistique pour calculer la distribution des orientations des fibres. Ainsi, l'orientation d'un grand nombre de fibres avec des orientations initiales couvrant la sphère unité est calculée à l'aide de l'équation de Jeffery, tout au long des lignes de courant associées, en utilisant une méthode de Runge-Kutta d'ordre 4. Les composantes des tenseurs $\underset{=}{a}$ et $\underset{\equiv}{=}$ dérivent des équations [9] et [10] :

$a_{11}=\sum_{i=1}^{N} \sin ^{2} \theta_{i} \cos ^{2} \beta_{i} / N, \quad a_{12}=\sum_{i=1}^{N} \sin ^{2} \theta_{i} \cos \beta_{i} \sin \beta_{i} / N$,
$a_{13}=\sum_{i=1}^{N} \sin \theta_{i} \cos \theta_{i} \cos \beta_{i} / N, \quad a_{22}=\sum_{i=1}^{N} \sin ^{2} \theta_{i} \sin ^{2} \beta_{i} / N, \ldots$.

$$
\begin{aligned}
& a_{1111}=\sum_{i=1}^{N} \sin ^{4} \theta_{i} \cos ^{4} \beta_{i} / N, \quad a_{1112}=\sum_{i=1}^{N} \sin ^{4} \theta_{i} \cos ^{3} \beta_{i} \sin \beta_{i} / N \\
& a_{1122}=\sum_{i=1}^{N} \sin ^{4} \theta_{i} \cos ^{2} \beta_{i} \sin ^{2} \beta_{i} / N, \quad a_{1222}=\sum_{i=1}^{N} \sin ^{4} \theta_{i} \cos \beta_{i} \sin ^{3} \beta_{i} / N \\
& a_{2222}=\sum_{i=1}^{N} \sin ^{4} \theta_{i} \sin ^{4} \beta_{i} / N
\end{aligned}
$$


où $\beta$ est l'angle entre l'axe $x$ et la projection de la fibre dans le plan de l'écoulement (plane $x-y)$, et $\theta$ est l'angle entre l'axe $z$ et elle-même.

\subsection{Procédure de résolution}

Dans cette section nous allons décrire sommairement la procédure de résolution. Les équations de conservation de la masse et de la quantité de mouvement, [1] et [2], respectivement, ainsi que l'équation constitutive de la suspension (équation [3]) sont normalisées en considérant la hauteur du domaine, $H$, et la vitesse moyenne du fluide, $U$. Le nombre de Reynolds est donc donné par $\operatorname{Re}=\rho U H / \eta$. Le domaine de calcul vient donné $\operatorname{par}\left(x^{*}, y^{*}\right) \in[0,10] \times[0,0.5]$ où seulement la moitié de la hauteur est considérée à cause de la symétrie. Pour plus de détails concernant le calcul adressez-vous à Chiba et al. (2001).

Dans la section d'entrée, un profil parabolique de vitesse est imposé, ainsi qu'un état d'orientation isotrope. Des fibres avec deux rapports de forme différents ont été considérées : $\lambda \approx \infty$ et $\lambda=10$, avec dans tous les cas le nombre de Reynolds $\operatorname{Re}=10$. Le paramètre $\phi \mu / \eta$ varie dans l'intervalle $2 \sim 10$. Nous avons ainsi montré l'incidence de ce paramètre dans l'évaluation de $a_{1111}$ dans le cas 3D (voir Azaiez et al., 2002, Fig. 27): le nombre de fibres à observer entre les angles 0 et $90^{\circ}$ dans la direction de $\theta$ doit être supérieur à 30 . Avec $N_{s}=30$, le nombre total de fibres considérées dans le calcul est de $N=2277$.

\subsection{Cinématique de l'écoulement et orientation des fibres}

La figure 6(a) illustre l'évolution de la vitesse tout au long de l'axe de symétrie de 1 'écoulement pour les deux types de fibres. La variation de vitesse la plus importante a lieu pour $x^{*}=0.9 \sim 0.975$. Cependant, l'effet de l'introduction de fibres disparaît progressivement en aval de l'écoulement pour des fibres à rapport de forme infini. L'effet des fibres à rapport de forme fini $(\lambda=10)$ est clairement mis en évidence dans cette figure, où l'on peut apprécier des variations plus faibles. De plus, pour des fibres à rapport de forme infini, l'évolution de la vitesse tout au long de l'axe de symétrie de l'écoulement devient très lisse, par rapport à l'évolution obtenue pour $\lambda=10$ qui montre des vagues, prouvant qu'un régime établi d'écoulement existe seulement pour des fibres à rapport de forme infini.

Les profils de vitesses $u^{*}$ (composante dans la direction $x$ ) sont montrés dans la figure 6 (b) pour $\lambda=10$. Par rapport aux résultats pour des fibres à rapport de forme infini (Azaiez et al., 2002, Fig. 30), le profil de $u^{*}$ pour $\lambda=10$ montre, tout au long du domaine, des vagues produites par la rotation continue des fibres. 


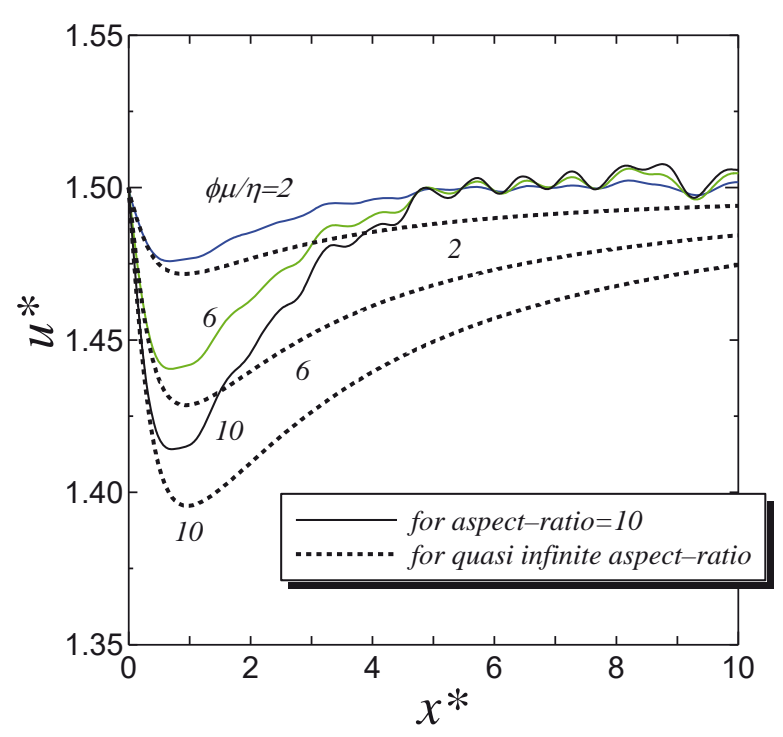

(b)

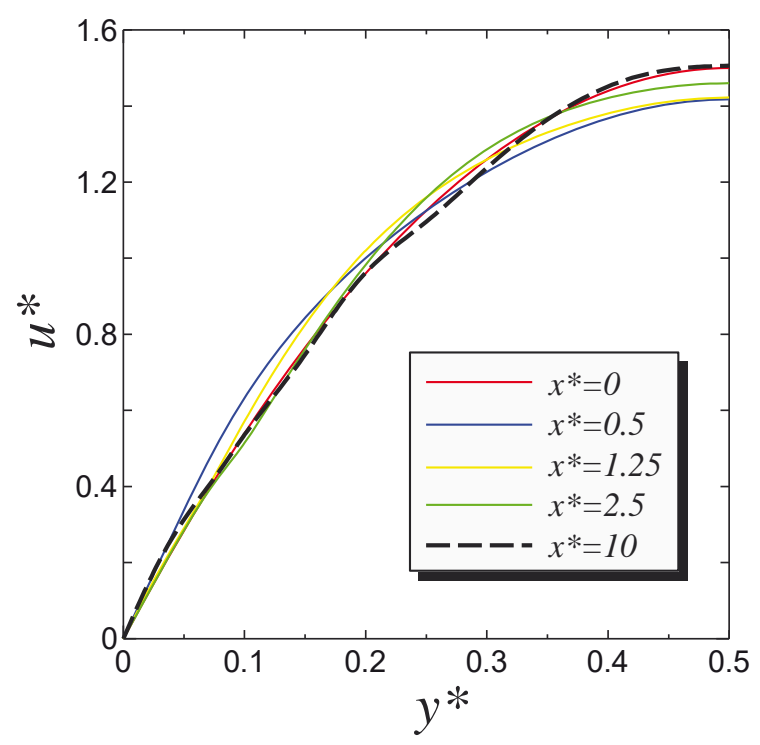

(a)

Figure 6. Profils de vitesses sur l'axe de symétrie de l'écoulement d'une suspension de fibres ( $\mathrm{Re}=10$ ), avec un état d'orientation 3D; (a) vitesses dans le cas des fibres avec un rapport de forme infini (trait discontinu) et des fibres à rapport de forme fini $(\lambda=10)$ (trait continu); (b) profils de vitesses dans le cas d'une suspension avec $\lambda=10$

Les figures 7(a) et (b) illustrent l'évolution de l'orientation des fibres (projection des ellipsoïdes sur le plan $x-y$ ) tout au long des lignes de courant à proximité des parois, pour des fibres à rapport de forme infini et pour des fibres avec $\lambda=10$ respectivement. Dans le cas des fibres à rapport de forme infini, la solution est lisse, contrairement au cas $\lambda=10$ où la rotation continue des fibres donne lieu à des solutions qui évoluent dans l'espace. Dans le cas $\lambda \approx \infty$ l'orientation évolue, comme prévu, d'un état isotrope à l'alignement des fibres avec l'écoulement. Dans la figure 7(b), on peut observer que les ellipsoïdes tournent périodiquement, et elles le font de plus en plus rapidement en s'approchant des parois où les gradients de cisaillement sont maximums. De plus, nous avons vérifié que les projections des ellipsoïdes sur le plan $x-z$ sont significativement moins importantes que leurs projections sur le plan $x-y$ (plane de l'écoulement) malgré les rotations qui ont lieu. Nous pouvons donc conclure que l'écoulement établi aura lieu seulement dans le cas d'une suspension de fibres à rapport de forme infini, quand un état d'orientation isotrope est imposé à l'entrée. 


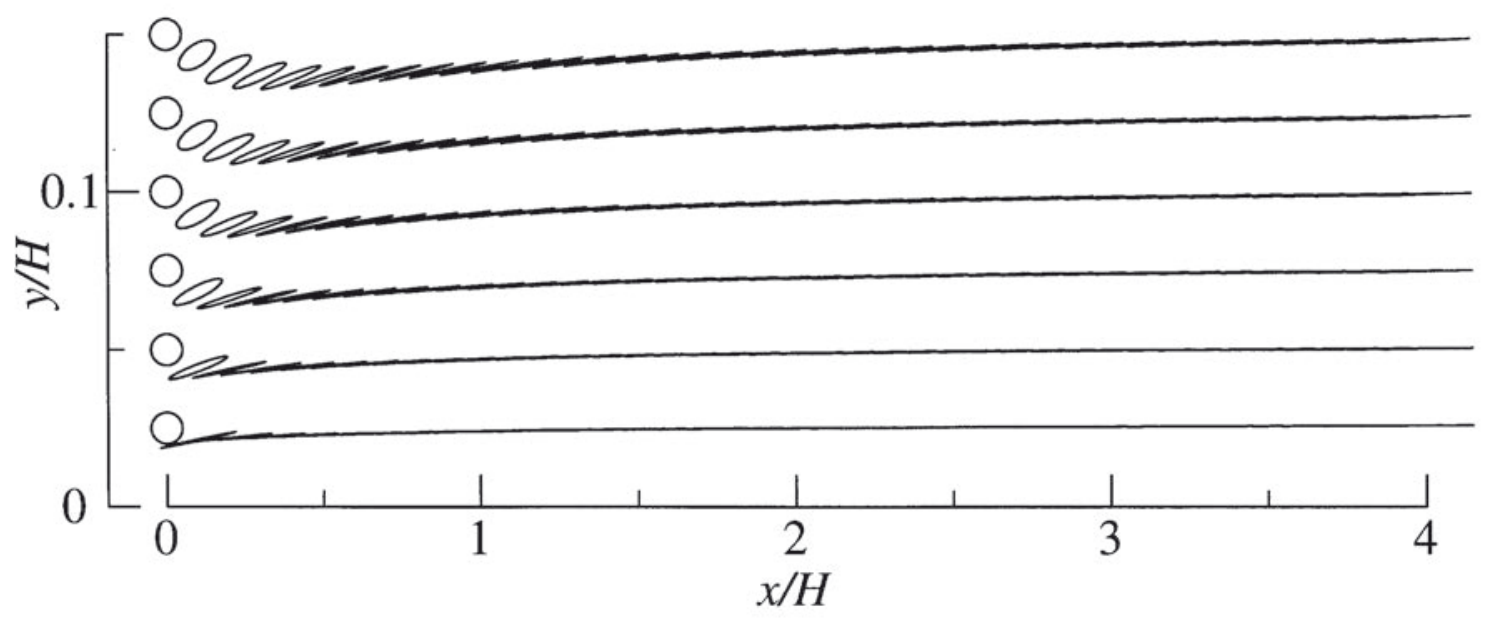

(a)

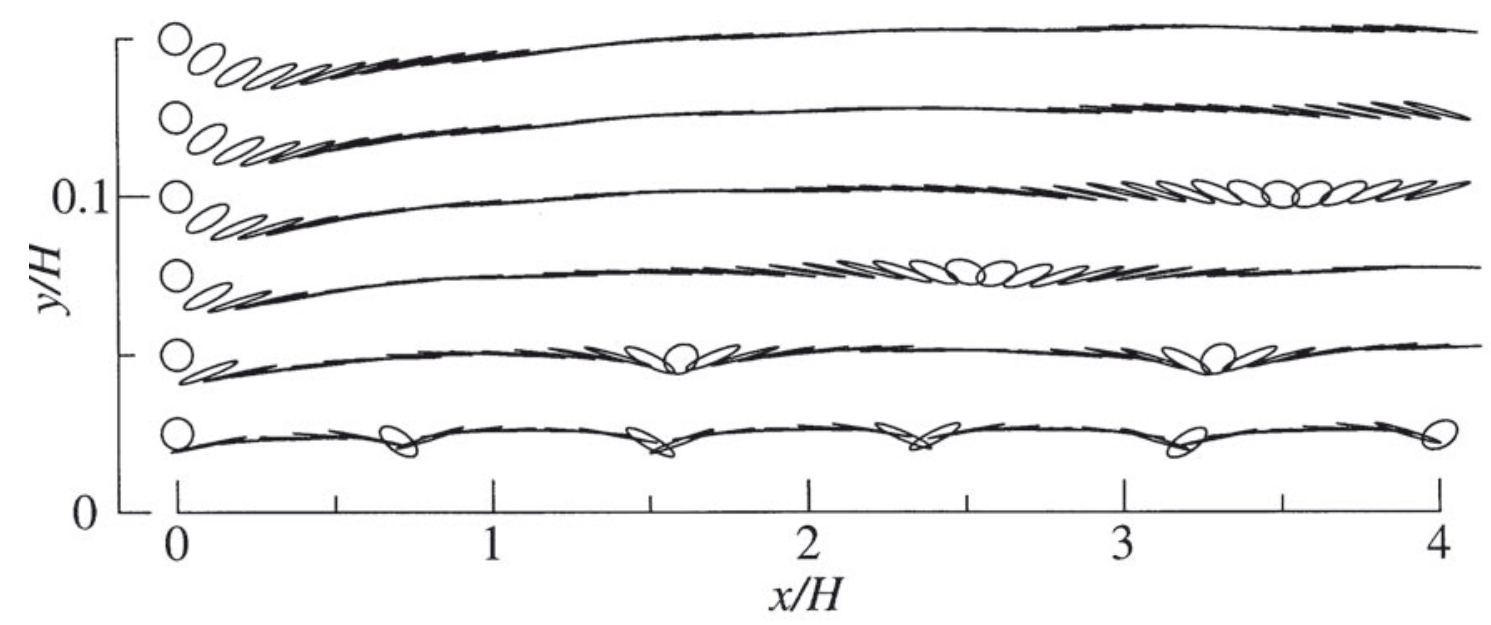

(b)

Figure 7. Evolution de l'orientation des fibres - représentées par des ellipsoïdes (en réalité leurs projections sur le plan $x-y$ ) - tout au long des lignes de courant, pour $\operatorname{Re}=10$ et $\phi \mu / \eta=10$; (a) $\lambda \approx \infty$; (b) $\lambda=10$.

\subsection{Champ de contraintes}

La contrainte de cisaillement $\tau_{x y}^{*}$ et la différence de contraintes normales $\tau_{x x}^{*}-\tau_{y y}^{*}$ sont représentées pour $0 \leq x^{*} \leq 4$ dans les figures 8 et 9 , respectivement, où la contrainte a été normalisée de la manière suivante: $\tau_{i j}^{*}=\tau_{i j} /(\eta U / H)$. Dans le cas d'un fluide Newtonien, $\tau_{x y}^{*}$ varie linéairement d'une valeur de 6 sur la paroi jusqu'à la valeur 0 sur l'axe de symétrie de l'écoulement, la différence de contraintes normales $\tau_{x x}^{*}-\tau_{y y}^{*}$ étant nulle partout.

Par contre, la distribution de contraintes devient plus complexe dans le cas d'une suspension de fibres courtes, principalement à proximité de la frontière d'entrée, où l'évolution des orientations est la plus forte. $\tau_{x y}^{*}$, tout comme $\tau_{x x}^{*}-\tau_{y y}^{*}$ augmente 
significativement par rapport aux valeurs associées à l'écoulement Newtonien à proximité de la frontière d'entrée et des parois. De plus, la valeur de $\tau_{x x}^{*}-\tau_{y y}^{*}$ devient très négative à proximité de l'axe de symétrie de l'écoulement; c'est la conséquence de la décélération du fluide introduit avec un profil parabolique, qui entraîne une forte compression sur l'axe, contrairement aux proximités des parois où le fluide est étiré.

L'effet de l'orientation 3D sur la contrainte est montrée dans les figures 8(a)-(b) et 9(a)(b) pour des fibres avec $\lambda \approx \infty$ : le degré d'isotropie pour un état d'orientation $3 \mathrm{D}$ est plus grande que pour le cas plan, et par conséquent, les variations des contraintes dans le cas 3D seront plus faibles que dans le cas $2 \mathrm{D}$. Les valeurs maximales de $\tau_{x y}^{*}$ sont 14.4 et 11.1 pour le cas $2 \mathrm{D}$ et $3 \mathrm{D}$ respectivement. Les valeurs extrêmes de $\tau_{x x}^{*}-\tau_{y y}^{*}$ sont $13.9,-3.44$ et 10.2 , 1.82, respectivement. De l'autre côté, les distributions de $\tau_{x y}^{*}$ et de $\tau_{x x}^{*}-\tau_{y y}^{*}$ deviennent de plus en plus complexes en s'approchant des parois, dans le cas $\lambda=10$, à cause de la rotation des fibres. Dans les figures 7(b), 8(c) et 9(c), nous pouvons constater que la contrainte de cisaillement augmente significativement là où les fibres tournent. Pour la différence de contraintes normales, elle décroît juste avant l'endroit où les fibres tournent, pour augmenter tout de suite après la rotation, à cause de la compression (en amont) et l'extension (en aval) du fluide.

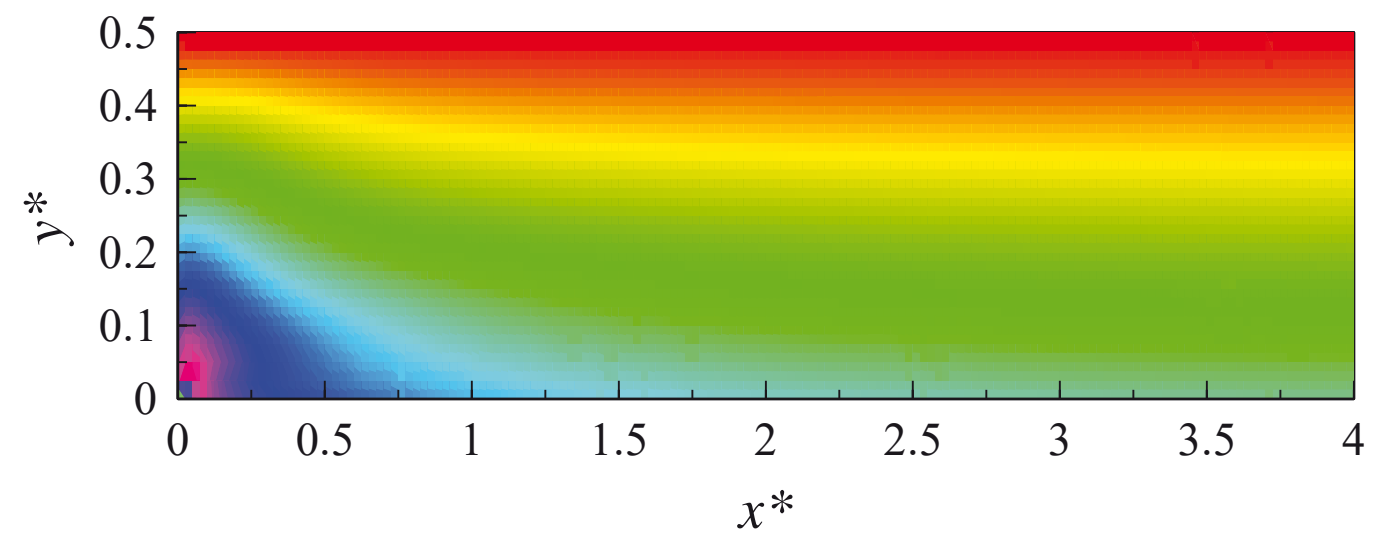

(a)

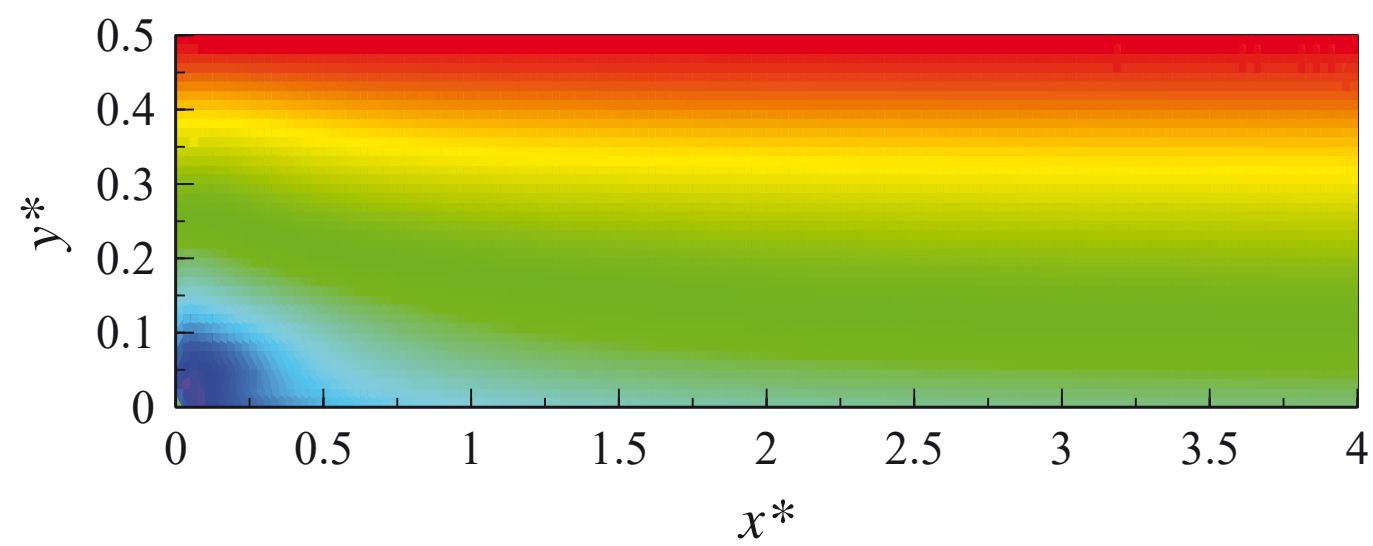




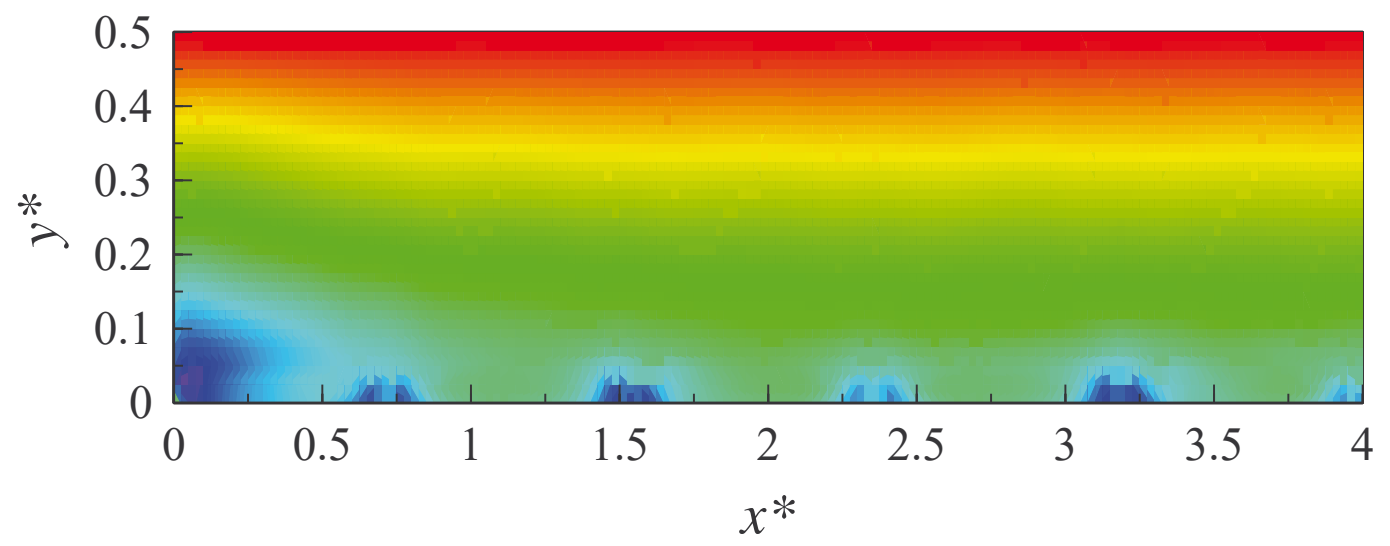

(c)

Figure 8. Distribution des contraintes $\tau_{x y}^{*}$ pour $\operatorname{Re}=10$ et $\phi \mu / \eta=10$; (a) $\lambda=\infty$ et orientation $2 \mathrm{D}$; (b) $\lambda=\infty$ et orientation $3 \mathrm{D}$; (c) $\lambda=10$ et orientation $3 \mathrm{D}$.

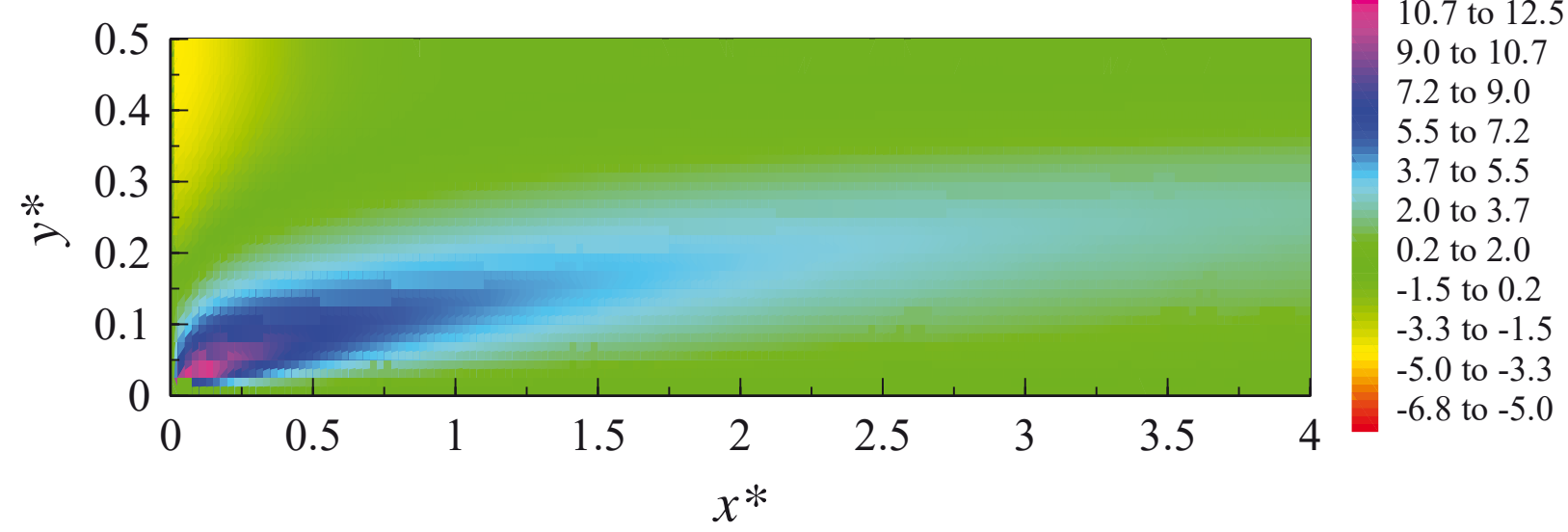

(a)

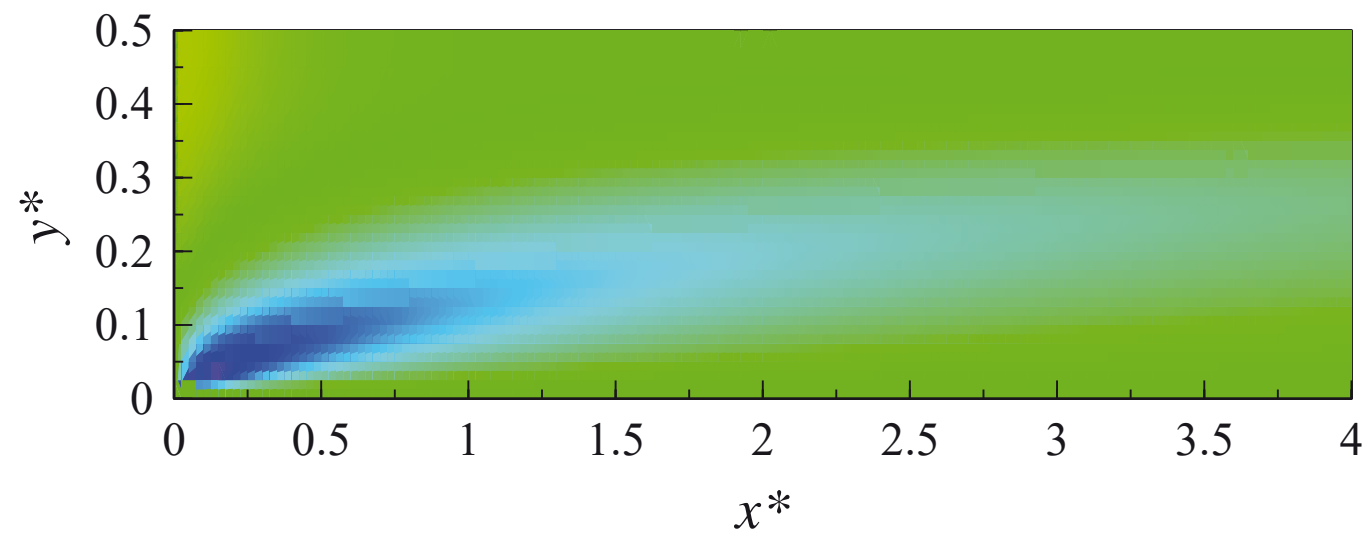

(b) 


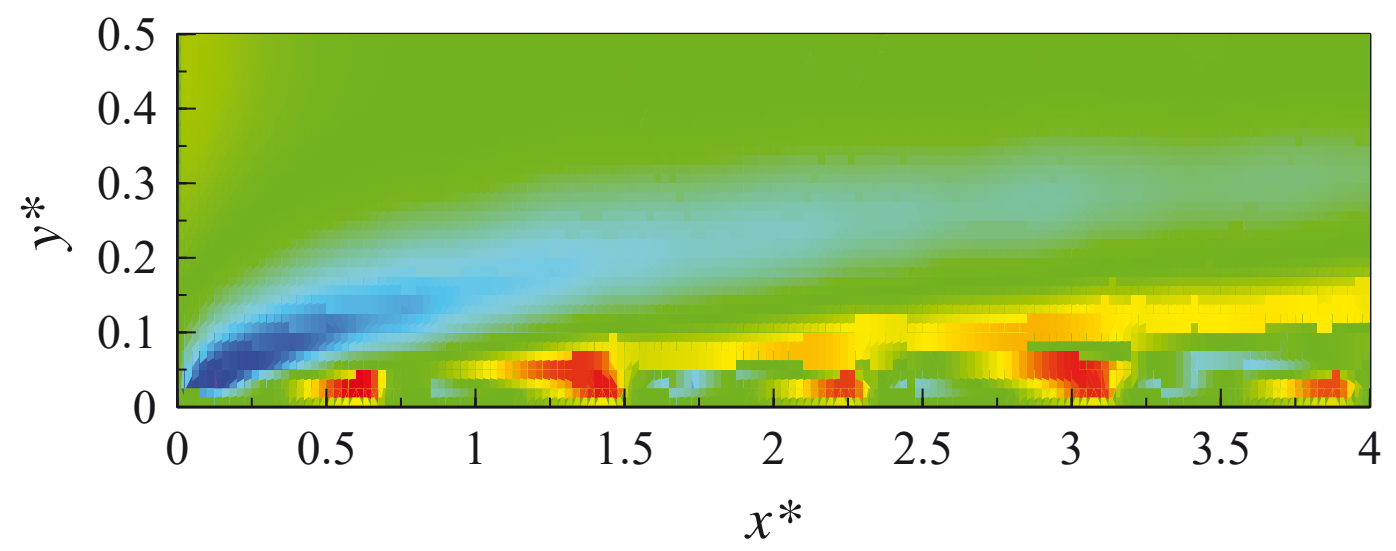

(c)

Figure 9. Distribution de la différence de contraintes normales $\tau_{x x}^{*}-\tau_{y y}^{*}$ pour $\operatorname{Re}=10$ et $\phi \mu / \eta=10$; (a) $\lambda=\infty$ et orientation $2 \mathrm{D}$; (b) $\lambda=\infty$ et orientation $3 \mathrm{D}$; (c) $\lambda=10$ et orientation 3D.

\subsection{Conclusions}

L'anisotropie induite par l'écoulement a été bien identifiée à proximité de la frontière d'entrée du fluide. La cinématique de l'écoulement et la distribution des contraintes présentent de grandes différences par rapport aux solutions associées à l'écoulement Newtonien. Cependant, pour des fibres à rapport de forme infini, l'effet de l'introduction de fibres disparaît progressivement, contrairement à ce qu'on peut observer dans le cas des suspensions de fibres à rapport de forme fini, à cause de la rotation continue des fibres. Ainsi, dans ce dernier cas et pour certains états d'orientation initiale (isotrope dans le cas traité ici), un régime d'écoulement établi n'est pas atteint.

\section{Références}

Abdul-Karem, T., Binding, D.M., Sindelar, M., « Contraction and expansion flows of nonNewtonian fluids », Compos. Manuf., vol. 4, 1993, p. 109-116.

Ahmed, A., Alexandrou, A.N., « Unsteady flow of semi-concentrated suspensions using finite deformation tensors », J. Non-Newtonian Fluid Mech., vol. 55, 1994, p. 115-136.

Ait-Kadi, A., Grmela, M., « Modelling the rheological behaviour of fibre suspensions in viscoelastic media », J. Non-Newtonian Fluid Mech., vol. 53, 1994, p. 65-81.

Altan, M.C., Güceri, S.I., Pipes, R.P., « Anisotropic channel flow of fiber suspensions », J. Non-Newtonian Fluid Mech., vol. 42, 1992, p. 65-83.

Altan, M.C., Rao, B.N., « Closed-form solution for the orientation field in a center-gated disk », J. Rheol., vol. 39, 1995, p. 581-599.

Ausias, G., Agassant, J.F., Vincent, M., Lafleur, P.G., Lavoie, P.A., Carreau, P.J., «Rheology of short glass fiber reinforced polypropylene », J. Rheol., vol. 36, 1992, p. 525-542. 
Ausias, G., Agassant, J.F., Vincent, M., « Flow and fiber orientation calculations in reinforced thermoplastic extruded tubes », Intern. Polym. Proc., vol. 9, 1994, p. 51-59.

Azaiez, J., « Constitutive equations for fiber suspensions in viscoelastic media », J. NonNewtonian Fluid Mech., vol. 66, 1996, p. 35-54.

Azaiez, J., Chiba, K., Chinesta, F., Poitou, A., « State-of-the-Art on Numerical Simulation of Fiber-Reinforced Thermoplastic Forming Process », Arch. Comput. Meth. Engng., vol. 9, 2002, p. 141-198.

Baloch, A., Webster, M.F., " A computer simulation of complex flows of fiber suspensions », Comput. Fluids, vol. 24, 1995, p. 135-151.

Chiba, K., Nakamura, K., Boger, D.V., « A numerical solution for the flow of dilute fiber suspensions through an axisymmetric contraction », J. Non-Newtonian Fluid Mech., vol. 35, 1990, p. 1-14.

Chiba, K., Yasuda, K., Nakamura, K., « Numerical solution of fiber suspension flow through a parallel plate channel by coupling flow field with fiber orientation distribution », J. Non-Newtonian Fluid Mech., vol. 99, 2001, p. 145-157.

Chono, S., Makino, M., " Numerical simulation of fiber suspension flow between parallel plates », Trans-B. Mach. Soc. Japan, vol. 61, 1995, p. 3190-3196.

Chung, S.T., Kwon, T.H., « Numerical simulation of fiber orientation in injection molding of short-fiber-reinforced thermoplastics », Polym. Eng. Sci., vol. 35, 1995, p. 604-618.

Dinh, S.M., Armstrong, R.C., "A rheological equation of state for semiconcentrated fiber suspensions », J. Rheol., vol. 28 , 1984, p. 207-227.

Ericksen, J.L., «Anisotropic fluids », Arch. Rat. Mech. Anal., vol. 4, 1960, p. 231-237.

Ghosh, T., Grmela, M., Carreau, P.J., « Rheology of short fiber filled thermoplastics», Polym. Compos., vol. 16, 1995, p. 144-153.

Lipscomb, G.G., Ph.D dissertation, University of California, Berkeley, 1986.

Lipscomb, G.G., Denn, M.M., Hur, D.H., Boger, D.V., « The flow of fiber suspensions in complex geometries », J. Non-Newtonian Fluid Mech., vol. 26, 1988, p. 297-325.

Phan-Thien, N., Graham, A.L., « A new constitutive model for fibre suspensions: flow past a sphere », Rheol. Acta, vol. 30, 1991, p. 44-57.

Ranganathan, S., Advani, S.G., « A simultaneous solution for flow and fiber orientation in axisymmetric diverging radial flow », J. Non-Newtonian Fluid Mech., vol. 47, 1993, p. 107-136.

Rosenberg, J., Denn, M.M., Keunings, R., « Simulation of non-recirculating flows of dilute fiber suspensions », J. Non-Newtonian Fluid Mech., vol. 37, 1990, p. 317-345. 